\title{
MARTINGALE FORMULATION OF ERGODIC THEOREMS 1
}

\section{MEYER JERISON}

1. Introduction. The similarity between martingale convergence theorems and ergodic theorems has been noticed for a long time. This similarity goes beyond the mere statements of the theorems; it extends to their proofs and their applications as well. A discussion of this matter may be found in [9].

In this note, it is shown ( $\$ 3$ ) that individual ergodic theorems can be formulated as statements about the convergence a.e. of martingales with decreasing index on infinite measure spaces. This is not to say that the ergodic theorems follow from the standard martingale convergence theorems, for the latter refer only to finite measure spaces. And although some generalizations of these theorems to spaces of infinite measure are known, none of them is broad enough to include the martingales that arise in the statement of ergodic theorems.

The martingale convergence theorem for finite measure spaces does yield the convergence a.e. of certain subsequences of the averages of the ergodic theorem, as is shown in $\$ 4$. In $\$ 5$, a theorem of convergence of martingales in $L_{p}$ is derived from a very general mean ergodic theorem.

2. Martingales on an infinite measure space. Throughout this paper, $(U, \mathcal{u}, \mu)$ will denote a totally $\sigma$-finite measure space. If $\mathcal{F}$ is a $\sigma$-subring of $\mathcal{u}$, we shall not distinguish, notationally, between the measure $\mu$ on $\mathfrak{u}$ and its restriction to $\mathcal{F}$.

2.1. Definition. ${ }^{2}$ Let $\mathcal{F}$ be a $\sigma$-subalgebra of $\mathcal{u}$ such that $(U, \mathcal{F}, \mu)$ is $\sigma$-finite, and let $x$ be a non-negative function, measurable with respect to $\mathcal{u}$, such that the measure

$$
\int_{A} x d \mu, \quad A \in \mathcal{F}
$$

defined on $(U, F)$ is $\sigma$-finite. The Radon-Nikodym derivative [7, p. 133] of this measure with respect to $\mu$ is called the conditional expectation of $x$ relative to $\mathcal{F}$ and is denoted by $E\{x \mid \mathcal{F}\}$. If, for an arbitrary function $x$, both $x^{+}=\sup (x, 0)$ and $x^{-}=(-x)^{+}$have conditional 1958.

Presented to the Society, September 1, 1955; received by the editors November 3 ,

1 The preparation of this paper was supported by the National Science Foundation.

${ }^{2}$ Conditional expectation is defined in [3] for a larger class of functions than that considered here. 
expectations relative to $\mathcal{F}$, then the conditional expectation of $x$ relative to $\mathcal{F}$ is defined as

$$
E\{x \mid \mathfrak{F}\}=E\left\{x^{+} \mid \mathfrak{F}\right\}-E\left\{x^{-} \mid \mathfrak{F}\right\} .
$$

Note that an integrable function has a conditional expectation relative to any $\sigma$-subalgebra. However, $x$ may have a conditional expectation even if $\int_{A} x d \mu$ is not always defined. This occurs when $\int_{U} x^{+} d \mu=\int_{U} x-d \mu=\infty$. In any case, if $E\{x \mid \mathcal{F}\}$ exists, it is defined to within a function that vanishes almost everywhere, and it is characterized by the conditions

2.2. (i) $E\{x \mid \mathcal{F}\}$ is measurable with respect to $F$, and

(ii) $\int_{A} E\{x \mid \mathfrak{F}\} d \mu=\int_{A} x d \mu$, for all $A \in \mathcal{F}$ for which the right member has meaning.

2.3. Definition. A family $\left(x_{n}\right)_{n \in J}$ of measurable functions on $(U, \mathcal{u}, \mu)$, indexed by some set $J$ of integers, is called a martingale if there exists a family $\left(\mathscr{F}_{n}\right)_{n \in J}$ of $\sigma$-subalgebras of $\mathcal{U}$ such that

$$
\digamma_{m} \subset F_{n} \text { and } x_{m}=E\left\{x_{n} \mid F_{m}\right\} \quad \text { whenever } m<n \text {. }
$$

It is often convenient to exhibit the family $\left(\mathcal{F}_{n}\right)$ by writing $\left(x_{n}, \mathfrak{F}_{n}\right)_{n \in J}$ to denote the martingale.

In this definition, the implicit assumption is made that all of the conditional expectations exist. In particular, it is assumed that $\left(U, \mathcal{F}_{n}, \mu\right)$ is $\sigma$-finite, for all $n \in J$.

In case the index set $J$ has a largest member $j$, then $x_{j}$ together with the family $\left(\mathcal{F}_{n}\right)$ determine the entire martingale, for we have $x_{n}=E\left\{x_{j} \mid \digamma_{n}\right\}$ for all $n \in J$. More generally, given an increasing family $\left(\mathcal{F}_{n}\right)_{n \in J}$ of $\sigma$-subalgebras, and a function $x$ all of whose conditional expectations exist, then the family $\left(E\left\{x \mid \mathcal{F}_{n}\right\}\right)_{n \in J}$ is a martingale. This follows from the formula

$$
E\left\{x \mid \mathcal{F}_{m}\right\}=E\left\{E\left\{x \mid \mathscr{F}_{n}\right\} \mid \mathcal{F}_{m}\right\} \quad(m<n)
$$

which is easy to verify.

Martingales on a finite measure space $(\mu(U)<\infty)$ are treated in detail in [4]. The most important theorems about such martingales are those concerning convergence. Thus, if $J$ is the set of positive integers, and if the sequence $\left(\int_{U}\left|x_{n}\right| d \mu\right)$ is bounded, then $\lim _{n \rightarrow \infty} x_{n}$ exists a.e. [4, p. 319]. This conclusion may be extended immediately to the case of $\sigma$-finite measure by applying the result to each of a countable number of members of $F_{1}$ of finite measure $[2$, p. 8]. The requirement of a bound on the integrals is also lightened to refer only to the integrals over the sets of finite measure. Thus, the functions need not even be integrable over all of $U$. 
We shall be interested in martingales that are indexed by the set of negative integers. For them, the following convergence theorem is valid $[4$, p. 328$]$ :

2.5. If $\left(x_{-n}\right)_{n \geq 1}$ is a martingale on $(U, \mathcal{u}, \mu), \mu(U)<\infty$, and $\int_{U}\left|x_{-1}\right| d \mu<\infty$, then $\lim _{n \rightarrow \infty} x_{-n}$ exists a.e.

A simple description of the limit can be given here. Let $\mathcal{F}_{-\infty}$ $=\bigcap_{n=1}^{\infty} \mathcal{F}_{-n}$. Then $\mathcal{F}_{-\infty}$ is clearly a $\sigma$-subalgebra, and it turns out that $\lim _{n \rightarrow \infty} x_{-n}=E\left\{x_{-1} \mid \mathcal{F}_{-\infty}\right\}$.

The hypothesis $\mu(U)<\infty$ can be dropped from this theorem, just as in the case of the martingales with increasing index, but for a different reason. Whereas the smallest $\sigma$-algebra, $\mathcal{F}_{1}$, that occurs in the case of increasing index is $\sigma$-finite by hypothesis, this is not true of the smallest $\sigma$-algebra, $\mathcal{F}_{-\infty}$, that occurs in the case of decreasing index. However, there exist $V, W$ in $\mathcal{F}_{-\infty}$ such that ${ }^{3} U=V \cup W$, where $V$ is a countable union of members of $\mathcal{F}_{-\infty}$ of finite measure, while any subset of $W$ that belongs to $\mathcal{F}_{-\infty}$ has measure 0 or $\infty$. Convergence of the martingale a.e. on $V$ is deduced immediately from 2.5 , and on $W$ from the following:

2.6. Let $\left(x_{-n}, \mathcal{F}_{-n}\right)_{n \geq 1}$ be a martingale, and suppose that each member of $\mathcal{F}_{-\infty}=\cap \mathcal{F}_{-n}$ has measure 0 or $\infty$. If $\int_{U}\left|x_{-1}\right| d \mu<\infty$, then $\lim _{n \rightarrow \infty} x_{-n}$ $=0$ a.e.

PROOF. Consider the extended real valued function $\bar{x}=\lim \sup _{n \rightarrow \infty} x_{-n}$. Clearly, $\bar{x}$ is measurable relative to each $\sigma$-ring $\mathcal{F}_{-n}$, and hence, relative to $\mathcal{F}_{-\infty}$. Thus, given a rational number $b>0$, the set $B$ $=\{u: \bar{x}(u) \geqq b\}$ belongs to $\mathcal{F}_{-\infty}$. By hypothesis, $\mu(B)=0$ or $\infty$. Suppose $\mu(B)=\infty$. Since $B \subset \bigcup_{n=1}^{\infty} A_{n}$, where

$$
A_{n}=\left\{u: \max _{1 \leqq k \leqq n} x_{-k}(u) \geqq b\right\},
$$

there exists $n$ such that $\mu\left(A_{n}\right)>b^{-1} \int_{U}\left|x_{-1}\right| d \mu$. Now, $\left(U, \mathcal{F}_{-n}, \mu\right)$ is totally $\sigma$-finite, and therefore, there exists $F \in \mathcal{F}_{-n}$ such that $\mu(F)<\infty$ and $\mu\left(F \cap A_{n}\right)>b^{-1} \int_{U}\left|x_{-1}\right| d \mu$. But, by $[4$, p. 314, Theorem 3.2], $b \mu\left(F \cap A_{n}\right) \leqq \int_{F \cap A_{n}} x_{-1} d \mu \leqq \int_{U}\left|x_{-1}\right| d \mu$, a contradiction. Thus, $\mu(B)$ $=0$. Since $b$ is an arbitrary positive rational number, we have $\bar{x}=\lim \sup _{n \rightarrow \infty} x_{-n} \leqq 0$ a.e. This result applied to the martingale $\left(-x_{-n}, \mathcal{F}_{-n}\right)$ yields $\lim \inf _{n \rightarrow \infty} x_{-n} \geqq 0$ a.e. Consequently, $\lim _{n \rightarrow \infty} x_{-n}=0$ a.e.

It is the integrability of $x_{-1}$ over all of $U$ that leads to the vanishing of the limit. In the case of martingales with increasing index, it was

${ }^{3}$ The measure algebra of $(U, \mathcal{u}, \mu)$ is a complete Boolean algebra in which the supremum of any family is the supremum of some countable subfamily $[7$, p. 169, (3) and (4) ]. Hence, a supremum, $V$, of the family of sets of finite measure in $\mathcal{F}_{-\infty}$ is a countable union of such sets. Take $W=U-V$. 
possible to relax the requirement of boundedness of the integrals by restricting it to each of a sequence of sets of finite measure whose union is $U$. In the present case, however, such a relaxation may lead to divergence of the martingale.

2.7. ExAmple. Let $(N, \mathfrak{N}, \nu)$ be the space of non-negative integers, the measure of each set being the number of its elements. Let $\mathcal{S}_{-n}$ be the smallest $\sigma$-algebra that contains the set $\{0,1, \cdots, n-1\}$ and all one-element sets $\{k\}$ with $k \geqq n$. Then every martingale $\left(x_{-n}, \mathcal{S}_{-n}\right)_{n \geqq 1}$ takes the form

$$
x_{-n}=E\left\{x \mid \mathcal{G}_{-n}\right\},
$$

where $x=x_{-1}$, and conversely, given any function $x$ on $N$ (i.e., any sequence of real numbers) the above defines a martingale. Moreover it is easy to check that conditions 2.2 (i) and (ii) for $E\left\{x \mid S_{-n}\right\}$ are satisfied by

$$
\begin{aligned}
x_{-n}(j) & =\frac{1}{n} \sum_{i=0}^{n-1} x(i) & 0 \leqq j<n \\
& =x(j) & j \geqq n .
\end{aligned}
$$

Thus, convergence of the martingale $\left(x_{-n}\right)$ is equivalent to convergence of the Cesaro means of the sequence $x$. This provides examples of martingales that diverge as well as convergent ones for which $\int_{N} x_{-1} d \nu$ does not exist.

3. Ergodic theorems. We show, in this section, that the individual ergodic theorems with discrete parameter are statements of convergence of suitable martingales on infinite measure spaces. We do this for two classes of ergodic theorems which include virtually all known ones (with a single discrete parameter).

On a totally $\sigma$-finite measure space $(S, \S, \sigma)$, let $L_{1}=L_{1}(S)$ denote the Banach space of integrable functions, with $\|x\|_{1}=\int_{S}|x(s)| d \sigma$, and let $T$ be a linear transformation of $L_{1}$ into itself. One class of ergodic theorems asserts the existence of $\lim _{n \rightarrow \infty} n^{-1} \sum_{l=0}^{n-1} T^{i} x(s)$, a.e. on $S$. Thus, Dunford and Schwartz [5, Theorem 3.6] proved it under the hypothesis $\|T x\|_{1} \leqq\|x\|_{1}$ and ess sup $|T x(s)| \leqq$ ess sup $|x(s)|$ for all $x \in L_{1}$.

Let $(U, \mathcal{u}, \mu)=(S \times N, \delta \times \mathfrak{T}, \sigma \times \nu)$, with $(N, \mathfrak{N}, \nu)$ as in 2.7. For each $n \geqq 1$, let $\mathcal{F}_{-n}$ be the family of all $A \in \mathcal{U}$ such that for all $s \in S$,

$$
(s, j) \in A \text { if and only if }(s, k) \in A \text {, whenever } j, k<n \text {. }
$$

In other words, $\mathcal{F}_{-n}$ is precisely the $\sigma$-algebra $\delta \times \mathcal{G}_{-n}$. Hence $\mathcal{F}_{-n}$ $\subset \mathcal{F}_{-m}$ if $n>m$, and if $f$ is a function on $U$ for which the conditional expectations exist, then $\left(E\left\{f \mid \mathcal{F}_{-n}\right\}\right)_{n \geq 1}$ is a martingale. 
3.1. Given $x \in L_{1}(S)$, let $f(s, k)=T^{k} x(s)$. Then, for each $n \geqq 1$, $E\left\{f \mid \mathcal{F}_{-n}\right\}$ exists and is equal to $f_{-n}$, where

$$
\begin{aligned}
f_{-n}(s, k) & =\frac{1}{n} \sum_{i=0}^{n-1} T^{i} x(s) & & \text { for } k<n \\
& =T^{k} x(s) & & \text { for } k \geqq n .
\end{aligned}
$$

Proof. To prove existence of the conditional expectation, it suffices to show that the indefinite integral of $|f|$ is a $\sigma$-finite measure on $\left(U, \mathcal{F}_{-n}\right)$. Now, $U=\cup_{k=0}^{\infty} U_{k}$, where $U_{k}=S \times\{k\}$, and the sets $\bigcup_{k=0}^{n-1} U_{k}, U_{n}, U_{n+1}, \cdots$, all belong to $\mathcal{F}_{-n}$. Since

$$
\int_{U_{k}}|f| d \mu=\int_{S}\left|T^{k} x\right| d \sigma=\left\|T^{k} x\right\|_{1}<\infty,
$$

the indefinite integral is $\sigma$-finite.

That $f_{-n}=E\left\{f \mid \mathcal{F}_{-n}\right\}$ is proved by verifying 2.2. Condition (i) is obviously satisfied by $f_{-n}$. To verify condition (ii), for given $A \in F_{-n}$, let $A^{\prime}=A \cap \bigcup_{k=0}^{n-1} U_{k}$ and $A^{\prime \prime}=A-A^{\prime}$. Then $A^{\prime}$ and $A^{\prime \prime}$ both belong to $\mathcal{F}_{-n}$. Since $f_{-n}$ agrees with $f$ on $A^{\prime \prime}, \int_{A^{\prime \prime}} f_{-n} d \mu=\int_{A^{\prime \prime}} f d \mu$. From the definition of $\mathcal{F}_{-n}$ and the fact that $A^{\prime} \subset \bigcup_{k=0}^{n-1} U_{k}$, it follows that $A^{\prime}=Q \times\{0,1, \cdots, n-1\}$, for some $Q \in \mathcal{S}$. Consequently,

$$
\int_{A^{\prime}} f d \mu=\sum_{i=0}^{n-1} \int_{Q} T^{i} x d \sigma .
$$

Now, for $k<n, f_{-n}(s, k)$ is independent of $k$, and hence

$$
\int_{A^{\prime}} f_{-n} d \mu=n \int_{A^{\prime} \cap U_{0}} f_{-n} d \mu=n \int_{Q} \frac{1}{n} \sum_{i=0}^{n-1} T^{i} x d \sigma .
$$

Thus, $\int_{A^{\prime}} f_{-n} d \mu=\int_{A^{\prime}} f d \mu$, and therefore, $\int_{A} f_{-n} d \mu=\int_{A} f d \mu$.

3.2. In the notation of 3.1, $\left(f_{-n}\right)_{n \geq 1}$ is a martingale, and $\left(f_{-n}\right)$ converges a.e. in $U$ if and only if $\left(n^{-1} \sum_{i=0}^{n-1} T^{i} x(s)\right)$ converges a.e. in $S$.

This follows directly from 3.1 and the preceding remarks, and exhibits the Dunford-Schwartz theorem, for example, as a statement of the convergence of certain martingales. We turn, next, to the ergodic theorem of Hurewicz [8] as generalized by Oxtoby [10].

3.3. Let $\phi$ be a one-one, measurable transformation of $a \sigma$-finite measure space $(S, \delta, \sigma)$; let $\tau$ be a finite signed measure on $S$ that is absolutely continuous ${ }^{5}$ with respect to $\sigma$; for $n=1,2, \cdots$, let

$$
\tau_{n}(B)=\sum_{k=0}^{n-1} \tau\left(\phi^{k} B\right), \quad \sigma_{n}(B)=\sum_{k=0}^{n-1} \sigma\left(\phi^{k} B\right), \quad(B \in \delta) ;
$$

${ }^{4}$ I.e., $\phi B$ and $\phi^{-1} B$ are measurable whenever $B$ is.

- Oxtoby eliminates the hypothesis of absolute continuity by using a more general notion of derivative. 
and let $x_{n}$ be the Radon-Nikodym derivative of $\tau_{n}$ with respect to $\sigma_{n}$. Then $\left(x_{n}(s)\right)$ converges to a finite limit except on a set $D$ such that $\sigma\left(\phi^{k} D\right)=0$ for $k=0,1, \cdots$.

The connection between this theorem and various generalized ergodic theorems is discussed in the papers cited above.

Our objective, again, is to construct a martingale whose convergence is equivalent to that of the sequence $\left(x_{n}\right)$. The space $(U, \mathcal{u}, \mu)$ will be, as before, $(S \times N, \delta \times \Re, \sigma \times \nu)$. The $\sigma$-subalgebra $\mathcal{F}_{-n}$ of $\mathcal{U}$ is defined as follows: $A \in \mathcal{F}_{-n}$ provided that for $k=0,1, \cdots, n-1$, and all $s \in S$,

$$
\left(\phi^{k} s, k\right) \in A \text { if and only if }(s, 0) \in A \text {. }
$$

3.4. Let $f(s, k)=x_{1}(s) \quad(s \in S, k \in N)$ (notation as in 3.3). Then $E\left\{f \mid \mathcal{F}_{-n}\right\}=f_{-n}$, where

$$
\begin{aligned}
f_{-n}(s, k) & =x_{n}\left(\phi^{-k} s\right) & 0 \leqq k<n \\
& =f(s, k) & k>n .
\end{aligned}
$$

The proof is quite analogous to that of 3.1.

3.5. In the notation of 3.3 and $3.4,\left(f_{-n}\right)_{n \geq 1}$ is a martingale. Convergence of this martingale almost everywhere on $U$ is equivalent to convergence of the sequence $\left(x_{n}(s)\right)$ except on a set $D$ such that $\sigma\left(\phi^{k} D\right)=0$ for $k=0,1,2, \cdots$.

Proof. From 3.4, we have $\lim _{n \rightarrow \infty} f_{-n}(s, k)=\lim _{n \rightarrow \infty} x_{n}\left(\phi^{-k} s\right)$ whenever either limit exists. Now, if $D$ is the set in $S$ where $\left(x_{n}(s)\right)$ diverges, then the set in $U$ where $\left(f_{-n}\right)$ diverges is precisely $\bigcup_{k=0}^{\infty} \phi^{k} D \times\{k\}$; and the measure $(\mu)$ of the latter set is 0 if and only if $\sigma\left(\phi^{k} D\right)=0$ for all $k$.

4. An application of the martingale convergence theorem. It seems most unlikely that a proof of an individual ergodic theorem might be based, in a straightforward way, upon the martingale convergence theorem 2.5. The latter can be used, however, to demonstrate the convergence of certain subsequences of the averages that occur in the ergodic theorem.

Here, $(S, \delta, \sigma)$ will be a totally finite measure space. We define $(U, \mathcal{U}, \mu)$ as the product space $(S \times I, \delta \times \mathfrak{g}, \sigma \times \lambda)$, where $(I, \mathfrak{g}, \lambda)$ is the Lebesgue measure space on the unit interval $[0,1)$. Let $\phi$ be a one-one, measure-preserving transformation of $S$ onto itself. We define, next, a sequence $\left(\mathcal{F}_{-n}\right)$ as follows: A set $A \in \mathcal{U}$ belongs to $\mathcal{F}_{-n}$ provided that for all $s \in S, 0 \leqq \alpha<2^{-n}$, and integers $k, 0 \leqq k<2^{n}$,

$$
\left(\phi^{k} s, \alpha+k \cdot 2^{-n}\right) \in A \text { if and only if }(s, \alpha) \in A \text {. }
$$

Given $x \in L_{1}(S)$, the relation 


$$
f(s, \alpha)=x(s) \quad(s \in S, \alpha \in I)
$$

defines a function $f \in L_{1}(U)$; in fact,

$$
\int_{U} f d \mu=\int_{S} \int_{I} x(s) d \lambda d \sigma=\int_{S} x d \sigma .
$$

4.1. $E\left\{f \mid \mathcal{F}_{-n}\right\}(s, \alpha)=2^{-n} \sum_{i=0}^{2^{n}-1} x\left(\phi^{i-j} s\right)$, where the integer $j$ is determined by $j \cdot 2^{-n} \leqq \alpha<(j+1) 2^{-n}$.

Proof. Let $f_{-n}$ denote the function on $U$ given by the right side of the equation. If $0 \leqq \alpha<2^{-n}$ and $k$ is an integer with $0 \leqq k<2^{n}$, then

$$
f_{-n}\left(\phi^{k} s, \alpha+k \cdot 2^{-n}\right)=2^{-n} \sum_{i=0}^{2^{n}-1} x\left(\phi^{i-k} \phi^{k} s\right)=f_{-n}(s, \alpha) .
$$

Since $f_{-n}$ is measurable with respect to $\mathcal{u}$, this identity implies that $f_{-n}$ is measurable with respect to $\mathcal{F}_{-n}$. We have only to prove that for each $A \in \mathcal{F}_{-n}, \int_{A} f_{-n} d \mu=\int_{A} f d \mu$. Let

$$
A_{k}=\left\{(s, \alpha) \in A: k \cdot 2^{-n} \leqq \alpha<(k+1) 2^{-n}\right\} \quad k=0,1, \cdots, 2^{n}-1 .
$$

Then the $A_{k}$ are pairwise disjoint, and $A=\mathrm{U}_{k} A_{k}$.

Now, from the definition of $\mathcal{F}_{-n}$, it follows that

$$
A_{k}=\left\{\left(\phi^{k} s, \alpha+k \cdot 2^{-n}\right):(s, \alpha) \in A_{0}\right\} .
$$

Moreover, since $\mu=\sigma \times \lambda$, and $\sigma$ is invariant under $\phi$ while $\lambda$ is invariant under translation in $I$, we have, for any $g \in L_{1}(U), \int_{A_{k}} g(s, \alpha) d \mu$ $=\int_{A_{0}} g\left(\phi^{k} s, \alpha+k \cdot 2^{-n}\right) d \mu$. Hence,

$$
\begin{aligned}
\int_{A} f d \mu & =\sum_{k=0}^{2^{n}-1} \int_{A_{k}} f d \mu=\sum_{k=0}^{2^{n}-1} \int_{A_{0}} f\left(\phi^{k} s, \alpha+k \cdot 2^{-n}\right) d \mu \\
& =\sum_{k=0}^{2^{n}-1} \int_{A_{0}} x\left(\phi^{k} s\right) d \mu ;
\end{aligned}
$$

and

$$
\begin{aligned}
\int_{A} f_{-n} d \mu & =\sum_{k=0}^{2^{n}-1} \int_{A_{0}} f_{-n}\left(\phi^{k} s, \alpha+k \cdot 2^{-n}\right) d \mu=2^{n} \int_{A_{0}} f_{-n}(s, \alpha) d \mu \\
& =2^{n} \int_{A_{0}} 2^{-n} \sum_{i=0}^{2^{n}-1} x\left(\phi^{i} s\right) d \mu .
\end{aligned}
$$

Therefore $f_{-n}=E\left\{f \mid \mathcal{F}_{-n}\right\}$.

4.2. Let $\phi$ be a one-one measure preserving transformation of $S$ onto itself. Given $x \in L_{1}(S)$, there exists a sequence $\left(j_{n}\right)_{n}$ in $N$ such that $\lim _{n \rightarrow \infty} 2^{-n} \sum_{i=0}^{2^{n}-1} s\left(\phi^{i-j_{n}} s\right)$ exists for almost all $s \in S$. 
Proof. By the martingale convergence theorem $2.5, \lim _{n \rightarrow \infty} f_{-n}(s, \alpha)$ exists a.e. in $U$. Hence, there exists $\alpha \in I$ such that $\lim _{n \rightarrow \infty} f_{-n}(s, \alpha)$ exists for almost all $s \in S$. Now, choose $j_{n}$ so that

$$
j_{n} \cdot 2^{-n} \leqq \alpha<\left(j_{n}+1\right) \cdot 2^{-n}
$$

for each $n \in N$.

4.3. REMARK. The set of all $\alpha$ with the property that $\left(f_{-n}(s, \alpha)\right)$ converges for almost all $s$ has measure 1 [7, p. 147, Theorem A]. Each such $\alpha$ determines, uniquely, a sequence $\left(j_{n}\right)$ for which the conclusion of 4.2 is valid. Thus, there exist uncountably many such sequences. Of course, the measure-theoretic argument used here cannot be used to single out any one of them.

4.4. REMARK. In the definition of the $\sigma$-subalgebras $\mathcal{F}_{-n}$, the sequence $\left(2^{n}\right)$ may be replaced by any sequence of integers $\left(m_{n}\right)$ such that $m_{n}$ is a divisor of $m_{n+1}$.

5. Convergence in mean. It is well known that mean ergodic theorems have been proved in more general settings than individual ergodic theorems. We shall show here that the mean convergence of martingales with decreasing index can be derived ${ }^{6}$ from the mean ergodic theorem of Alaoglu and Birkhoff [1, Theorem 9]. The statement of the theorem given below follows Eberlein [6, Theorem 3.1], although not in full generality.

5.1. Definition. Let $G$ be a semigroup of bounded linear transformations on a normed linear space $X$. A net (i.e., a directed system) $\left(T_{\alpha}\right)$ of linear transformations of $X$ into itself is called a system of almost invariant integrals for $G$ if

(i) for each $x \in X$ and all $\alpha, T_{\alpha} x$ belongs to the closed convex hull of $\{T x: T \in G\}$;

(ii) the transformations $T_{\alpha}$ are uniformly bounded;

(iii) for every $x \in X$ and $T \in G$,

$$
\lim _{\alpha}\left(T T_{\alpha} x-T_{\alpha} x\right)=\lim _{\alpha}\left(T_{\alpha} T x-T_{\alpha} x\right)=0 .
$$

5.2. MEAN ERGODIC THEOREM. If $\left(T_{\alpha}\right)$ is a system of almost invariant integrals for $G$, and if for a given $x \in X$, the net $\left(T_{\alpha} x\right)$ has a weak cluster point $y$, then $y=\lim _{\alpha} T_{\alpha} x$ (in norm).

This theorem will be applied to the space $L_{\alpha}(U)$ of functions $x$ on $U$ such that $\|x\|_{p}=\left(\int_{U}|x|^{p} d \mu\right)^{1 / p}<\infty$.

5.3. Let $\left(x_{-n}, \mathcal{F}_{-n}\right)_{n \geqq 1}$ be a martingale on a finite measure space $(U, \mathcal{u}, \mu)$. If, for some $p \geqq 1, x_{-1} \in L_{p}(U)$, then $x_{-n} \in L_{p}(U)$ for all $n$, and there exists $x_{-\infty} \in L_{p}(U)$ such that $\lim _{n \rightarrow \infty}\left\|x_{-n}-x_{-\infty}\right\|_{p}=0$.

${ }^{6} \mathrm{I}$ am indebted to J. L. Doob for calling this to my attention. 
Proof. For each $n$, define $T_{n}$ by setting $T_{n} x=E\left\{x \mid \mathcal{F}_{-n}\right\}\left(x \in L_{p}\right)$. Now, it follows from the definition of conditional expectation, that $\left|E\left\{x \mid \mathcal{F}_{-n}\right\}\right|^{p} \leqq E\left\{|x|^{p} \mid \mathcal{F}_{-n}\right\} \quad\left[4\right.$, p. 33]. This implies that $\left\|T_{n} x\right\|_{p}$ $\leqq\left(\int_{U} E\left\{|x|^{p} \mid \mathcal{F}_{-n}\right\} d \mu\right)^{1 / p}=\|x\|_{p}$, so that, in particular, $T_{n} x \in L_{p}$. Also, formula (2.4) becomes $T_{m} T_{n}=T_{n} T_{m}=T_{\max (m, n)}$. Consequently, the set $G=\left\{T_{n}\right\}_{n \geqq 1}$ is a semigroup of bounded linear transformations, and moreover, the sequence $\left(T_{n}\right)$ itself is a system of almost invariant integrals for $G$. Thus, 5.2 is applicable. In case $p>1$, any bounded set in $L_{p}$ has weakly compact closure, and hence, for each $x \in L_{p}$, the sequence $\left(T_{n} x\right)$ has a weak cluster point. In $L_{1}$, the set $\left\{T_{n} x\right\}_{n}$ still has weakly compact closure because the set is uniformly integrable [4, p. 311, Theorem 3.1 (iv)]. In either case, therefore, 5.2 asserts the existence of a limit, in the $L_{p}$ norm, of $\left(T_{n} x\right)$. In particular, the sequence of functions $x_{-n}=T_{n} x_{-1}$ converges.

5.4. REMARK. For $p>1$, the theorem and proof are valid also in case $\mu(U)=\infty$. This is not so, however, for $p=1$. Setting $x(0)=1$, $x(n)=0(n>0)$, in Example 2.7, yields a martingale that does not converge in $L_{1}$.

\section{REFERENCES}

1. L. Alaoglu and G. Birkhoff, General ergodic theorems, Ann. of Math. (2) vol. 41 (1940) pp. 293-309.

2. E. S. Andersen and B. Jessen, Some limit theorems on integrals in an abstract set, Danske Vid. Selsk. Mat.-Fys. Medd. vol. 22 no. 14 (1946).

3. Y. S. Chow, Thesis, University of Illinois, 1958.

4. J. L. Doob, Stochastic processes, New York, John Wiley and Sons, 1953.

5. N. Dunford and J. T. Schwartz, Convergence almost everywhere of operator averages, J. Rat. Mech. Anal. vol. 5 (1956) pp. 129-178.

6. W. F. Eberlein, Abstract ergodic theorems and weak almost periodic functions, Trans. Amer. Math. Soc. vol. 67 (1949) pp. 217-240.

7. P. R. Halmos, Measure theory, New York, D. Van Nostrand Co., 1950.

8. W. Hurewicz, Ergodic theorem without invariant measure, Ann. of Math. (2) vol. 45 (1944) pp. 192-206.

9. S. Kakutani, Ergodic theory, Proceedings of the International Congress of Mathematicians, Cambridge, 1950, vol. 2, pp. 128-142.

10. J. C. Oxtoby, On the ergodic theorem of Hurewicz, Ann. of Math. (2) vol. 49 (1948) pp. 872-884.

PuRdue University AND

The Institute for Advanced Study 